\title{
Analysis of Theme Evolution in the Novel Don Quijote de la Mancha -Additional Comment on Cervantes' Creative Intention
}

\begin{abstract}
Daichen $^{*}$
Department of Culture and Communication, Xichang College, Xichang, Sichuan province, China. 280792721@qq.com

ABSTRACT

The theme in the novel Don Quijote de la Mancha has always been conflicting for a long history. Referring the view of Manuel, the reason for the divergence lies in Cervantes's division of conscious creation and unconscious creation. The former clearly shows opposition and ridicule knight novels in his works, while the latter provides a wider space for explaining the theme of the novel. From the 17th century to the 19th century, researchers believed that the theme of the novel was to defend Christianity, criticize the real society, and eulogize idealism. In the 20 st century, researchers' interpretation of the theme of the novel is more unique and more diverse.
\end{abstract}

Keywords: Don Quijote de la Mancha, Cervantes, theme, diverse

\section{浅析小说《堂吉诃德》主题演变 兼论塞万提斯创作意图}

\section{代晨*}

西昌学院文化传媒学院，西昌，四川，中国

280792721@qq.com

\section{摘要}

关于小说《堂吉诃德》的主题, 历来众说纷纭。产生分歧的原因, 借用曼努埃尔的观点, 塞万提斯在创作时, 分为了有意识的创作和无意识的创作。前者在作品中明确表露出反对、嘲弄骑士小说，后者则为阐释小说主题 提供了更加广阔的空间。从 17 世纪到 19 世纪，研究者认为小说主题为捍卫基督教、批判现实社会、歌颂理想 主义。进入 20 世纪，研究者对小说主题的解读，视角更加独特，观点也更加的多元。

关键词: 《堂吉诃德》,塞万提斯, 主题, 多元

\section{1. 《堂吉诃德》文学地位与永恒魅力}

西班牙作家胡安・韦尔塔斯曾建议: 最好敦请国 王陛下下诏, 让国内 60 岁以下的人都学会读书、写 字, 然后阅读《堂吉诃德》。这一观点不足为奇, 相 比于胡安的 “高论”, 一些《堂吉诃德》的西班牙研 究者甚至将这部小说视为西班牙的《圣经》。时至今 日, 《堂吉诃德》不仅是西班牙文学的丰碑宝塔, 而 且早已位列世界经典文学行列。2002 年, 在瑞典文学
院等机构联合举办的一次民意测验中，来自 54 个国 家和地区的 100 位作家投票选举 “人类最佳文学作 品”, 《堂吉诃德》名列第一, 得票率高达 $50 \%$ 以上。

《堂吉诃德》的魅力源于作品的多个方面。这里, 笔者将着重分析作品在流传过程中的主题演变。为解 答相关问题, 还需追本溯源, 从塞万提斯的创作意图 入手。 


\section{2. 时代背景与创作意图}

翻阅《堂吉诃德》开头, 会发现塞万提斯借小说 作者友人之口, 道出了小说的创作意图 “把骑士小说 那些呼神唤鬼的谎言扫除干净”。小说结尾处, 塞万 提斯还借阿拉伯传记作者之口, 表示此书的宗旨就是 “引起世人厌恶虚妄荒诞的骑士小说”。开头和结尾 的呼应, 已将塞万提斯的创作意图表露无遗。

塞万提斯创作《堂吉诃德》并非突发奇想, 探析 背后的原因, 主要有两个。首先, 荒诞不经和谎话连 篇的骑士小说层出不穷。西班牙学者梅嫩德斯在《塞 万提斯的文学渊源》一文中分析了骑士小说兴盛的原 因：一是社会方面的原因, 包括骑士和宫廷的思想, 习俗, 惯例, 举止和实践的转变; 二是骑士小说占据 市场, 尽管大多数骑士小说艺术水准低下, 却具有娱 乐功能, 深受市民读者的喜爱。其次, 骑士小说的粗 制滥造, 让当时的一些人意识到了危害, 进行了抨击。 尤其在 1545 年举办的特兰托教务会议上, 有教士就 向骑士小说发起了攻击, 谓骑士小说和新教一样害人。

正是在此背景下, 塞万提斯创作了《堂吉诃德》。 小说中, 堂吉诃德原名为阿隆索 - 吉哈诺, 是居住在 拉曼恰的一位绅士，衣食无忧，整日沉溺骑士小说， 逐渐丧失理智, 进而效仿小说中的游侠骑士, 外出闯 荡, 幻想伸张正义、锄强扶弱、建功立业, 结果却处 处碰壁。

小说中, 塞万提斯采用了多种艺术手法, 以期达 到反对、嘲弄骑士小说的目的。这里, 笔者聚焦在 “戏 仿” 和 “消解” 两种艺术手法。在以往的骑士小说中, 主人公大多英俊潇酒、天生神力, 骑着高头大马, 携 带宝刀宝剑, 但在《堂吉诃德》中, 这一设定却遭到 了 “戏仿”。小说中, 担任骑士的堂吉诃德, 年过半 百, 干瘦瘦弱, 骑的马瘦成了皮包骨头, 武器也早已 布满了锈渍和霉斑, 不得不洗刷清洗。通过 “戏仿”, 完全颠覆了以往骑士小说中的人物形象, 给人滑稽、 可笑之感。

另外, 在骑士小说中, 爱情和战争可谓两大看点。 在小说第一部第 47 章中, 借小说人物教长之口, 为 读者呈现出以往骑士小说中的两大看点: 少年宝剑一 挥, 将巨人拦腰截断; 勇士单枪匹马, 击败了百万雄 兵; 女皇初见骑士, 不顾矝持投怀送抱。但在《堂吉 诃德》中, 战争和爱情被 “消解” 得平淡无奇, 乃至 无踪无影。战争中, 且不说堂吉诃德常被凡夫俗子打 得人仰马翻, 最讽刺的, 莫过于堂吉诃德深受骑士小 说的影响, 按图索瀷, 把风车当作巨人, 将羊群视为 军队。至于小说中的爱情, 有时是堂吉诃德一厢情愿, 认为对方深爱着自己; 有时是别人有意捉弄, 故意让 堂吉诃德难堪。尤其是堂吉诃德眼中美丽、高贵的心 上人杜尔西内亚, “消解” 之后, 无非是一个粗鄙的 村妇。小说结尾处, 堂吉诃德第三次出游被人打败, 回到家乡, 临死前幡然醒悟, 不仅认识到了骑士小说 的危害, 还长篇大论、振振有词的怒斥了骑士小说。 塞万提斯出色的艺术手法, 完全达到了反对、嘲弄骑
士小说的目的。以至于两个世纪后, 英国诗人拜伦在 《唐璜》中不由感叹: 塞万提斯把西班牙的骑士风笑 掉了, 一笑而把本国的元气摧毁无遗, 自那以后, 西 班牙很少英雄了。

拜伦观点未免夸张, 不过也引出一个问题: 倘若 只是单纯嘲弄骑士小说, 那为何《堂吉诃德》没有随 着骑士小说的销声匿迹而被人遗忘。由此可见，小说 主题绝不仅限于此。想要探析《堂吉诃德》的主题, 亦需钩沉索引, 先来再现最初读者的感受。

\section{3. 有意识创作与无意识创作}

1604 年，小说《堂吉诃德》出版后，迅速再版， 不仅成为了西班牙国内的畅销书，而且流传到了英、 法、意等国。这部小说给当时的读者带去了许多的欢 乐。根据 1662 年出版的《好王费利佩三世言行记》 记载, 国外费利佩三世有次在高处眺望, 见到远处有 位读书人拿着书本哈哈大笑，就对侍从说 “那个读书 人不是疯了, 就是被堂吉诃德的故事逗乐了”, 侍从 前去调查, 读书人果然在读《堂吉诃德》。

当时的读者, 并不认为《堂吉诃德》是一部严肃、 深遂之作, 相反, 被视为不登大雅之堂的遣闷、逗乐 之作。在民间庆祝活动中, 堂吉诃德、桑丘和杜尔西 内亚也被看作滑稽、可笑之人，被人们当作逗乐的小 丑, 到处演出。在欢乐和逗笑之间, 小说主题也被认 定为反对、嘲弄骑士小说。当时审阅《堂吉诃德》的 书检官马尔克斯 - 托雷斯一语道破小说主题: 作者旨 在扫除空洞无物、谎话连篇、流布甚广、茶毒无穷的 骑士书。小说流传半个多世纪后, 1665 年, 出版商马 特奥・巴斯蒂达在致友人信中也表露了自己观点, 认 为《堂吉诃德》用它的嘲弄揭露了骑士小说害人匪浅 的夸夸其谈, 从而解救了沉溺骑士小说的读者。

小说中构思的嘲讽骑士小说的情节和不断涌现 的批评、质疑骑士小说的声音, 让反对骑士小说的主 题得以彰显, 即便是后来的许多研究者, 也继承和认 同了这一观点。限于篇幅, 笔者不能一一列举, 不过 不妨将目光聚焦在 1780 年。这一年，西班牙皇家语 言学院推出了它的第一个学院版《堂吉诃德》, 该版 附有维森特院士的文章, 认为作品具有 “根治骑士文 学的毛病”。这一认定, 可视为官方和主流的权威结 论。

尽管如此, 但一些研究者并没有亦步亦趋认定小 说主题为反对、嘲弄骑士文学。毕竟作者创作意图和 读者接受程度无法全部契合。但如何绕开塞万提斯给 小说的定位和书中大量批评、质疑骑士小说的内容, 从而给小说更多的生机和活力, 一直是后来研究者需 要解决的一大难题。

到了 1875 年, 研究者曼努埃尔乾坤独断, 在出 版的《西班牙和美洲的光明》一书中，认为塞万提斯 在创作《堂吉诃德》时, 进行了有意识创作和无意识 创作, 前者表现出 “对骑士文学以及中世纪的骑士理 
想竭尽嘲弄、批评之能事”，后者则是塞万提斯不曾 预想、不曾设计、在无意识中创作的，是 “永恒的《堂 吉诃德》”。在曼努埃尔提出该观点之前, 研究者们已 经对《堂吉诃德》主题进行了不同解读, 曼努埃尔的 贡献在于赋予这些观点 “合法依据”, 并且为之后研 究者提供了更多阐释空间。

\section{4. 《堂吉诃德》主题的传统解读}

从 17 世纪到 19 世纪, 围绕着《堂吉诃德》的主 题论述，下列三方面具有一定的代表性:

\section{1. 捍卫基督教}

1571 年, 西班牙、威尼斯、罗马教廷组成联军, 共同抵抗奥斯曼土耳的入侵, 双方在勒班托海域爆发 大战, 最终以联军的获胜而告终。塞万提斯也参加这 次海战, 并光荣负伤、失去左臂。在回国途中又遭遇 海盗劫持, 被风禁在阿尔及尔, 直到 5 年后才凑足赎 金, 重获自由。《堂吉诃德》中穿插的战俘的故事, 即是塞万提斯自身经历的写照—一即便深陷异教徒 的囚笼, 也要想方设法、矢志不渝的回到基督教世界。 除了自身经历, 塞万提斯在小说中, 还处处表现了劝 人向善, 赞成驱赶摩尔人 (异教徒), 肯定基督教价 值观等。这不仅让海涅将塞万提斯称为 “罗马教会的 忠诚儿子”, 而且导致一些研究者认为《堂吉诃德》 主题是宣扬捍卫基督教。1 861 年, 特奥多米罗 - 伊巴 涅斯出版《《<堂吉诃德・德・拉曼恰>在 19 世纪》一 书, 意在论证小说的基督教思想, 认为其所以成为经 久不衰的经典, 在于艺术地体现了永恒的基督教精神。 类似观点, 还有弗朗西斯科 -纳瓦雷特主教认为的《堂 吉诃德》具有 “反对败坏基督教道德、天主教信仰的 言行”。

\section{2. 批判现实社会}

塞万提斯的一生, 可以说见证了西班牙帝国的衰 落, 加上自身命途多多, 重获自由后, 回国担任了收 税员, 却因存放税款的钱庄破产, 再次深陷图圄; 且 长期生活在社会底层。塞万提斯丰富的经历和阅历, 有意或无意的反映在了小说中, 《堂吉诃德》也就成 为了解 16 世纪西班牙社会的百科全书。因而有研究 者认为小说是塞万提斯的 “自传”, 意在批判现实社 会。19 世纪 50 年代, 西班牙学者拉蒙・安特盖拉提 出了 “双重目的” 说, 认为塞万提斯创作 《堂吉诃德》 目的, 首先是对世界的批评, 其次才是反对骑士小说, 明确表示 “骑士小说只是《堂吉诃德》的一个切入口, 他由此对许多时弊进行了针砭”。学者何塞 - 斯巴尔 比也认为塞万提斯创作 《堂吉诃德》的动因是面对社 会的不公正和不公平，呼唤更多具有良知、信仰和正 义的堂吉诃德, 共同将社会所有的歪风邪气和沉疴流 弊一扫而光。此外, 一些研究者还认为小说将矛头对 准了人性。费尔南多 - 卡斯特罗认为《堂吉诃德》 “实 际上针对普遍的人性弱点”。

\section{3. 歌须理想主义}

小说中, 堂吉诃德曾直言 “老天把我生在这黑铁 时代, 是为了在世上恢复黄金时代, 就是人们常说的 光明时代”。但心怀崇高理想的堂吉诃德, 却处处碰 壁, 前后三次出游, 都铩羽而归, 这也反映出理想和 现实的冲突。这一点, 引起了许多后来者的共鸣。19 世纪初期, 德国哲学家谢林将堂吉诃德界定为浪漫主 义的不朽的典型, 明确表示小说主题是现实与理想的 斗争。谢林认为小说第一部, 表达了英雄的理想遭遇 了现实世界、现实环境的狙击; 小说第二部表达了主 人公进入了理想世界, 却发现与之冲突的正是理想本 身。同时期的海涅在德文版精印《堂吉诃德》序言中 不仅满怀深情回忆自己阅读小说时具有的感同身受 和无比同情, 而且认为堂吉诃德的理想是想让未来赶 早在当今出现, 虽然失败了, 但那种激昂奋发的热情, 却值得肯定。19 世界 60 年代, 屠格涅夫发表了《哈 姆莱特与堂吉诃德》的演讲, 高度赞扬了堂吉诃德全 身心浸透着对理想的忠诚, 为实现理想, 准备牺牲自 己的生命。笔者认为, 关于理想和现实的冲突, 正是 《堂吉诃德》长盛不衰的重要原因, 每一代的读者, 都能从中找到共鸣。小说结尾值得回味: 向现实低头 服输的是好人阿隆索 - 吉哈诺, 堂吉诃德只是暂时隐 退, 或者说早已将精神发扬光大, 激励了无数的后起 之秀。

\section{5. 《堂吉诃德》主题的多元解读}

进入 20 世纪, 从现代主义到后现代主义, 从结 构主义到解构主义, 从接受美学到文化批评, 批评方 式更加多样化, 解读视角更加多元化。限于篇幅, 对 《堂吉诃德》的主题研讨, 笔者选取了三个值得注意 的观点。

20 世纪 50 年代, 弗拉基米尔 -纳博科夫受邀在 哈佛大学讲学, 当着数百名学生的面, 将《堂吉诃德》 撕毁。纳博科夫反常举动背后有充分的观点支撑。纳 博科夫认为, 该书 “构成了一部以残酷性为主题的货 真价实的百科全书”。纳博科夫首先列举了小说第一 部折磨肉体的残酷性事实, 包括堂吉诃德被打的头破 血流、门牙飞落、失去半只耳朵等, 其次列举了小说 第二部折磨精神的残酷性表现, 主要包括公爵夫妇及 其奴仆对堂吉诃德、桑丘无休无止的捉弄。纳博科夫 无法容忍塞万提斯对人物遭遇痛苦时的津津乐道, 故 认为《堂吉诃德》是有史以来写下的最难以容忍、最 缺乏人性的书之一。

仅看上述内容, 很容易让人产生误解: 纳博科夫 对《堂吉诃德》深恶痛绝、全盘否认。事实上, 纳博 科夫并不否认堂吉诃德, 反而给予这一文学人物深刻 的理解和同情。究其原因, 或许流亡在外的纳博科夫 从越挫越勇、一往无前的堂吉诃德那里获得了生活的 力量和信心。此外, 纳博科夫创作的小说, 也深受《堂 吉诃德》的影响。其代表作《洛丽塔》就采用了 “流 
浪冒险” 的叙述方式, 小说主人公驾驶汽车, 穿越美 国东西部, 为读者展现了美国的社会风貌。

到了 20 世纪 80 年代, 也产生了重要的观点。米 兰 - 昆德拉在《受到诋毁的塞万提斯遗产》一文中指 出: 《堂吉诃德》并非关于理想主义的批评或颂扬, 而是一种“对被遗忘了的存在进行探寻”。具体而言, 昆德拉认为堂吉诃德能够随心所欲探索敞开的世界, 代表了一种自由探索的精神。随着历史和时代的发展, 这种精神日益萎缩。在巴尔扎克笔下, 人物生活在五 光十色的城市, 世界不再敞开; 在福楼拜笔下, 包法 利夫人的世界被日常琐事包裹, 梦和梦想的重要性增 加了; 到了卡夫卡笔下, 人物 $\mathrm{K}$ 被超现实力量牢牢 掌控, 只能一刻不停地想着土地测量或法律审判。昆 德拉将《堂吉诃德》和欧洲经典文学做了对比, 认为 堂吉诃德具有的探索精神, 正是欧洲艺术的伟大之处, 从而更加肯定了《堂吉诃德》。值得一提的, 20 世纪 90 年代, 布鲁姆在《西方正典》中, 借用了乌纳穆诺 的观点, 认为堂吉诃德是生存的探索者, 导致堂吉诃 德疯癫和产生疯癫的原因, 是“永不熄灭的生存渴望”。 这一观点, 和昆德拉的相比, 可谓异曲同工。

至于女性主义对《堂吉诃德》的主题分析, 笔者 选择了莫尼克 - 乔利迪在《从女性视角看〈堂吉诃德〉 的色情指设》中的观点。该文首先借用了卡尔维诺观 点一一任何文学都是色情的; 其次, 该文发现小说一 些独特女性时不时表现出有意或无意与骑士对话, 愿 意或无意成为骑士的梦中情人, 从而探讨当骑士的性 意念转变为堂吉诃德面对任何一位女士所发生的行 为动力时, 这些性意念是如何来影响小说女性人物性 格和语言特征。该文的分析未免有些牵强附会, 但这 也从一个侧面证明了《堂吉诃德》的包罗万象和常读 常新。

\section{6. 结论}

进入 21 世纪, 《堂吉诃德》依旧光彩夺目, 在世 界各地长盛不衰, 仅国内翻译的版本, 就有好几十种。 关于《堂吉诃德》的主题, 可谓仁者见仁, 智者见智, 未有定论。这也不难理解, 因为阅读《堂吉诃德》, 好比弹奏一架毒立的竖琴, 不同时代的读者都能从中 弹奏出满足自身需求和契合时代共鸣的旋律。这也反 映出《堂吉诃德》具有的广度和深度, 无愧为一部经 典、伟大的文学作品。

不过, 当下国内读者热衰阅读《堂吉诃德》, 主 要原因, 恐怕还在于从小说人物的疯癫和背时中, 激 发出长埋内心深处的理想和信仰, 以此来对抗现实生 活中的物欲膨胀和资本泛滥, 从而寻找到人生真正的 意义和价值。

\section{REFERENCES}

[1] Cervantes, Dong Yansheng (Translated in 2019), Don Quijote de la Mancha, Beijing Yanshan Publisher, Beijing
[2] Chen Zhongyi, (2011) Estudios sobre la historia de estudios cervantinos, Yilin Publisher, Nanjing

[3] Chen Qijia, Song Hui, (2014) Ideal Community and Illusion of Individual Freedom —-An Allegorical Reinterpretation of Knight Errantry in Don Quixote, Journal of Renmin University of China, 28(03):106113

[4] Nabokov, (2007) Lecture on Don Quixote, SDX Joint Publishing Company

[5] McGrath Michael J. ( 2020 ) Don Quixote and Catholicism:RereadingCervantineSpirituality [M].Purdue University Press 